\title{
Coefficient inequalities for certain starlike and convex functions
}

\author{
Sushil Kumar ${ }^{1}$ (D), Asena Çetinkaya ${ }^{* 2}$ (D) \\ ${ }^{1}$ Bharati Vidyapeeth's College of Engineering, Delhi-110063, India \\ ${ }^{2}$ Department of Mathematics and Computer Science, İstanbul Kültür University, İstanbul, Turkey
}

\begin{abstract}
In this paper, we consider two Ma-Minda-type subclasses of starlike and convex functions associated with the normalized analytic function $\varphi_{N e}(z)=1+z-z^{3} / 3$ that maps an open unit disk onto the Nephroid shaped bounded domain in the right-half of the complex plane. We investigate convolution and quasi-Hadamard product properties for the functions belonging to such classes. In addition, we compute best possible estimates on third order Hermitian-Toeplitz determinant and non-sharp estimates on certain third order Hankel determinants for the starlike functions associated with the interior region of Nephroid.
\end{abstract}

Mathematics Subject Classification (2020). 30C45, 30C50

Keywords. starlike functions, convex functions, nephroid, convolution properties, quasi-Hadamard product properties, Hermitian-Toeplitz and Hankel determinants

\section{Introduction}

The coefficient inequalities of the normalized analytic univalent functions yield it's geometric properties related information. Let $\mathbb{D}=\{z \in \mathbb{C}:|z|<1\}$ denotes the open unit disk and let $\mathcal{A}$ be the class of all analytic functions $f$ of the form

$$
f(z)=z+\sum_{n=2}^{\infty} a_{n} z^{n}
$$

defined in $\mathbb{D}$ and normalized by the conditions $f(0)=0$ and $f^{\prime}(0)=1$. Denote by $\mathcal{S}$ the subclass of $\mathcal{A}$ containing all the univalent functions in $\mathbb{D}$. Let $\Omega$ be the family of analytic functions $w$ satisfying the conditions $w(0)=0,|w(z)|<1$ for all $z \in \mathbb{D}$. If $f$ and $g$ are analytic functions in $\mathbb{D}$, then we say $f$ is subordinate to $g$, written as $f \prec g$, if there exists a function $w \in \Omega$ such that $f=g \circ w$. In particular, if $g \in \mathcal{S}$, the equivalence condition $f \prec g \Leftrightarrow f(0)=g(0)$ and $f(\mathbb{D}) \subset g(\mathbb{D})$ holds [8]. The function $f \in \mathcal{A}$ is starlike if $f(\mathbb{D})$ is starlike with respect to the origin and the function $f \in \mathcal{A}$ is convex if $f(\mathbb{D})$ is convex. In terms of subordination, the function $f \in \mathcal{A}$ is starlike and convex if and only if the subordination relations $z f^{\prime}(z) / f(z) \prec(1+z) /(1-z)$ and $z f^{\prime \prime}(z) / f^{\prime}(z) \prec 2 z /(1-z)$ for

\footnotetext{
*Corresponding Author.

Email addresses: sushilkumar16n@gmail.com (S. Kumar), asnfigen@hotmail.com (A. Çetinkaya)

Received: 08.08.2020; Accepted: 06.09.2021
} 
all $z \in \mathbb{D}$ respectively hold. Several subclasses of the starlike and convex functions were studied by many authors $[13,15,29,34-36]$ in the literature.

Using the concept of subordination, Ma and Minda [28] introduced and studied the unified classes $\mathcal{S}^{*}(\varphi)$ and $\mathcal{C}(\varphi)$ of starlike and convex functions, where $\varphi$ is the analytic function satisfying $\operatorname{Re}(\varphi(z))>0$ for all $z \in \mathbb{D}$. These classes contain various subclasses of starlike and convex functions. In recent past, several Ma-Minda-type classes of starlike and convex functions have been introduced and studied by various authors $[16,23,37,38]$. In this paper, we consider two subclasses $\mathcal{S}_{N e}^{*}$ and $\mathcal{C}_{N e}$ of Ma-Minda classes $\mathcal{S}^{*}(\varphi)$ and $\mathcal{C}(\varphi)$ respectively which are associated with the analytic function $\varphi_{N e}(z)=1+z-z^{3} / 3$ that is univalent, starlike with respect to 1 and maps $\mathbb{D}$ onto a Nephroid shaped bounded symmetric region with respect to real axis in the right-half plane. Analytically, these classes are defined as

$$
\mathcal{S}_{N e}^{*}=\left\{f \in \mathcal{S}: \frac{z f^{\prime}(z)}{f(z)} \prec \varphi_{N e}(z)\right\} \text { and } \mathcal{C}_{N e}=\left\{f \in \mathcal{S}: 1+\frac{z f^{\prime \prime}(z)}{f^{\prime}(z)} \prec \varphi_{N e}(z)\right\}
$$

for all $z \in \mathbb{D}$. Recently, these classes were introduced by Wani and Swaminathan [40]. They studied several properties of these classes such as the structural formula, growth and distortion theorems, Fekete-Szegö functionals, radius estimates [41] and subordination results.

If $f, g \in \mathcal{A}$, where $f$ is given by (1.1) and $g$ is given by $g(z)=z+\sum_{n=2}^{\infty} b_{n} z^{n}$, then the convolution or Hadamard product of $f$ and $g$, denoted by $f * g$, is defined by

$$
f(z) * g(z)=(f * g)(z)=z+\sum_{n=2}^{\infty} a_{n} b_{n} z^{n} .
$$

It is noted that if $g(z)=z /(1-z)$, then $f * g=f$ and if $g(z)=z /(1-z)^{2}$, then $f * g=z f^{\prime}$ for all $f \in \mathcal{A}$. Further, let $\mathcal{T}$ be the class of analytic functions with negative coefficients of the form

$$
f(z)=a_{1} z-\sum_{n=2}^{\infty} a_{n} z^{n}, \quad\left(a_{1}>0 ; a_{n} \geq 0\right)
$$

defined in $\mathbb{D}$. For the functions, $f$ defined by $(1.2)$ and $g(z)=b_{1} z-\sum_{n=1}^{\infty} b_{n} z^{n}$, the quasi-Hadamard product (or convolution) is given by

$$
f(z) * g(z)=a_{1} b_{1} z-\sum_{n=2}^{\infty} a_{n} b_{n} z^{n} .
$$

The quasi-Hadamard of two or more functions were defined by Owa [30] and Kumar [19]. Let the functions $f_{i}(i=1, \ldots, m)$ and $g_{j}(j=1, \ldots, s)$ of the form

$$
\begin{aligned}
& f_{i}(z)=a_{1, i} z-\sum_{n=2}^{\infty} a_{n, i} z^{n}, \quad\left(a_{1, i}>0 ; a_{n, i} \geq 0\right) \\
& g_{j}(z)=b_{1, j} z-\sum_{n=2}^{\infty} b_{n, j} z^{n}, \quad\left(b_{1, j}>0 ; b_{n, j} \geq 0\right)
\end{aligned}
$$

be analytic in $\mathbb{D}$. Denote by $h$ the quasi-Hadamard product $f_{1} * f_{2} * \ldots * f_{m} * g_{1} * g_{2} * \ldots * g_{s}$ is defined by

$$
h(z)=\left\{\prod_{i=1}^{m} a_{1, i} \prod_{j=1}^{s} b_{1, j}\right\} z-\sum_{n=2}^{\infty}\left\{\prod_{i=1}^{m} a_{n, i} \prod_{j=1}^{s} b_{n, j}\right\} z^{n} .
$$

In 2000, Hossen [12] established certain results related to quasi-Hadamard product for $p$-valent starlike and $p$-valent convex functions. Aouf [3] proved a theorem concerning to quasi-Hadamard product for certain analytic functions. Using uniformly starlikeness and uniformly convexity, Breaz and El-Ashwah [5] studied quasi-Hadamard product between some $p$-valent and uniformly analytic functions with negative coefficients. 
Hankel and Hermitian-Toeplitz determinants have important role in various branches of pure and applied mathematics. Let $\left\langle a_{k}\right\rangle_{k>1}$ denotes a sequence of coefficients of the normalized analytic function $f \in \mathcal{A}$. The coefficient estimates of normalized univalent functions in the disk $\mathbb{D}$ give many useful information regarding the geometric properties. For instance, the estimate on second coefficient of the function $f \in \mathcal{S}$ yields the growth and distortion theorems. This idea inspires researchers to determine the estimates on the coefficient functionals such as the Hermitian-Toepltiz and Hankel determinants. For $q, n \in \mathbb{N}$, the Hankel determinant of order $n$ associated with the sequence $\left\langle a_{k}\right\rangle_{k \geq 1}$ is defined by

$$
H_{q}^{(n)}(f):=\operatorname{det}\left\{a_{n+i+j-2}\right\}_{i, j}^{q}, \quad 1 \leq i, j \leq q, a_{1}=1 .
$$

For the functions $f \in \mathcal{S}$ and $f \in \mathcal{S}^{*}$, Hankel determinants were discussed initially by Pommerenke [31,32]. Later, Hayman (1968) [11] computed the best possible bound $\kappa n^{1 / 2}$ on Hankel determinant $\left|H_{2, n}(f)\right|$ for general univalent functions, where $\kappa$ as an absolute constant. In 2013, authors [26] determined sharp estimates on second Hankel determinant for Ma-Minda starlike and convex functions. In 2010, Babalola [4] first computed bounds on the third Hankel determinant for analytic functions with bounded-turning as well as starlike and convex functions. Later on, Zaprawa [43] obtained improved bounds for third order Hankel determinant obtained by Babalola [4] but these bounds were not sharp. Kowalczyk et al. [17] established sharp inequality $\left|H_{3}^{(1)}(f)\right| \leq 4 / 135$ for convex functions. Recently, Kumar et al. [22] improved certain existing bound on the third Hankel determinant for some classes of close-to-convex functions. For recent results on third Hankel determinant, see $[10,24,25,39]$. Hankel determinants are closely related to HermitianToeplitz determinants $[18,42]$. The third order Hermitian-Toeplitz determinant $T_{3,1}(f)$ for the function $f \in \mathcal{A}$ is given by

$$
\left|T_{3,1}(f)\right|:=2 \operatorname{Re}\left(a_{2}^{2} \overline{a_{3}}\right)-2\left|a_{2}\right|^{2}-\left|a_{3}\right|^{2}+1 .
$$

The sharp estimates on certain symmetric Toeplitz determinants were evaluated for univalent functions and typically real functions by Ali et al. [2]. Further, the best possible lower and upper bounds for the second and third-order Hermitian-Toeplitz determinants are estimated over the classes of starlike and convex functions of order $\alpha$ [7]. Jastrzȩbski [14] computed best possible upper and lower bounds of second and third order HermitianToeplitz determinants for some close-to-star functions. Recently, Kumar and Kumar [21] investigate sharp upper and lower bounds on third order Hermitian-Toeplitz determinant for the classes of strongly starlike functions.

Motivated by the above stated research work, second section provides convolution properties of the classes $\mathcal{S}_{N e}^{*}$ and $\mathcal{C}_{N e}$. Further, certain results associated with quasi-Hadamard product for such classes are established in Section 3. In the last section, we obtain best possible lower and upper bounds on the third-order Hermitian-Toeplitz determinant for starlike functions in the class $\mathcal{S}_{N e}^{*}$. In addition, non-sharp estimates on third-order Hankel determinants $H_{3}^{(1)}(f), H_{3}^{(2)}(f)$ and $H_{3}^{(3)}(f)$ for the functions $f$ belonging to the class $\mathcal{S}_{N e}^{*}$ are also computed.

\section{Convolution properties}

In view of the work done in $[6,9]$, we derive convolution properties of the classes $\mathcal{S}_{\mathrm{Ne}}^{*}$ and $\mathcal{C}_{N e}$. We first begin with necessary and sufficient convolution conditions of the class $\mathcal{S}_{N e}^{*}$

Theorem 2.1. The function $f$ defined by (1.2) is in the class $\mathcal{S}_{N e}^{*}$ if and only if

$$
\frac{1}{z}\left[f(z) * \frac{z-L z^{2}}{(1-z)^{2}}\right] \neq 0
$$

for all $L=\frac{3+3 e^{i \theta}-e^{3 i \theta}}{3 e^{i \theta}-e^{3 i \theta}}$, where $\theta \in[0,2 \pi]$ and also $L=1$. 
Proof. Suppose the function $f \in \mathcal{S}_{N e}^{*}$, then we have

$$
\frac{z f^{\prime}(z)}{f(z)} \prec 1+z-\frac{z^{3}}{3} .
$$

Since the function $z f^{\prime}(z) / f(z)$ is analytic in $\mathbb{D}$, it follows $f(z) \neq 0, z \in \mathbb{D}^{*}=\mathbb{D} \backslash\{0\}$; that is, $(1 / z) f(z) \neq 0$ and this is equivalent to the fact that $(2.1)$ holds for $L=1$. In view of relation $(2.2)$, we have

$$
\frac{z f^{\prime}(z)}{f(z)}=\frac{3+3 w(z)-w^{3}(z)}{3}
$$

where $w \in \Omega$. The expression (2.3) is equivalent to

$$
\frac{z f^{\prime}(z)}{f(z)} \neq \frac{3+3 e^{i \theta}-e^{3 i \theta}}{3}
$$

so that

$$
\frac{1}{z}\left[3 z f^{\prime}(z)-\left(3+3 e^{i \theta}-e^{3 i \theta}\right) f(z)\right] \neq 0 .
$$

Since we have convolution relations $f(z) * \frac{z}{1-z}=f(z)$ and $f(z) * \frac{z}{(1-z)^{2}}=z f^{\prime}(z)$, then expression (2.5) is written as

$$
\frac{1}{z}\left[f(z) *\left(\frac{3 z}{(1-z)^{2}}-\frac{\left(3+3 e^{i \theta}-e^{3 i \theta}\right) z}{(1-z)}\right)\right] \neq 0 .
$$

Therefore, we have

$$
\frac{e^{3 i \theta}-3 e^{i \theta}}{z}\left[f(z) * \frac{z-\frac{3+3 e^{i \theta}-e^{3 i \theta}}{3 e^{i \theta}-e^{3 i \theta}} z^{2}}{(1-z)^{2}}\right] \neq 0,
$$

which completes the necessary part of Theorem 2.1 .

Conversely, because assumption (2.1) holds for $L=1$, it follows that $(1 / z) f(z) \neq 0$ for all $z \in \mathbb{D}$, hence the function $\psi(z)=z f^{\prime}(z) / f(z)$ is analytic in $\mathbb{D}$, and it is regular at $z=0$ with $\psi(0)=1$. Since it was shown in the first part of the proof that assumption (2.1) is equivalent to (2.4), we have

$$
\frac{z f^{\prime}(z)}{f(z)} \neq \frac{3+3 e^{i \theta}-e^{3 i \theta}}{3}
$$

and if we denote

$$
\varphi_{N e}(z)=\frac{3+3 z-z^{3}}{3}
$$

relation $(2.7)$ shows that the simply connected domain $\psi(\mathbb{D})$ is included in a connected component of $\mathbb{C} \backslash \varphi_{N e}(\partial \mathbb{D})$. Using the fact $\psi(0)=\varphi_{N e}(0)$ together with the univalence of the function $\varphi_{N e}$, it follows that $\psi \prec \varphi_{N e}$, which represents (2.2). Thus, $f \in \mathcal{S}_{N e}^{*}$ which completes the proof of Theorem 2.1.

Theorem 2.2. A necessary and sufficient condition for the function $f$ defined by (1.2) to be in the class $\mathcal{S}_{\mathrm{Ne}}^{*}$ is that

$$
a_{1}-\sum_{n=2}^{\infty} \frac{3-3 n+3 e^{i \theta}-e^{3 i \theta}}{3 e^{i \theta}-e^{3 i \theta}} a_{n} z^{n-1} \neq 0 .
$$

Proof. From Theorem 2.1, $f \in \mathcal{S}_{N e}^{*}$ if and only if

$$
\frac{1}{z}\left[f(z) * \frac{z-L z^{2}}{(1-z)^{2}}\right] \neq 0
$$


for all $L=\frac{3+3 e^{i \theta}-e^{3 i \theta}}{3 e^{i \theta}-e^{3 i \theta}}$ and also $L=1$. The left-hand side of $(2.10)$ is written as

$$
\begin{aligned}
\frac{1}{z}\left[f(z) *\left(\frac{z}{(1-z)^{2}}-\frac{L z^{2}}{(1-z)^{2}}\right)\right] & =\frac{1}{z}\left\{z f^{\prime}(z)-L\left(z f^{\prime}(z)-f(z)\right)\right\} \\
& =a_{1}-\sum_{n=2}^{\infty}(n(1-L)+L) a_{n} z^{n-1} \\
& =a_{1}-\sum_{n=2}^{\infty} \frac{3-3 n+3 e^{i \theta}-e^{3 i \theta}}{3 e^{i \theta}-e^{3 i \theta}} a_{n} z^{n-1}
\end{aligned}
$$

which completes the desired proof.

We next determine coefficient estimate for a function of form (1.2) to be in the class $\mathcal{S}_{N e}^{*}$.

Theorem 2.3. If the function $f$ defined by (1.2) satisfies the following inequality

$$
\sum_{n=2}^{\infty}(3 n-1)\left|a_{n}\right| \leq 2 a_{1}
$$

then $f \in \mathcal{S}_{N e}^{*}$.

Proof. According to the expression(2.9), a simple computation gives

$$
\begin{aligned}
\left|a_{1}-\sum_{n=2}^{\infty} \frac{3-3 n+3 e^{i \theta}-e^{3 i \theta}}{3 e^{i \theta}-e^{3 i \theta}} a_{n} z^{n-1}\right| & \geq a_{1}-\sum_{n=2}^{\infty}\left|\frac{3-3 n+3 e^{i \theta}-e^{3 i \theta}}{3 e^{i \theta}-e^{3 i \theta}}\right|\left|a_{n}\right| \\
& =a_{1}-\sum_{n=2}^{\infty} \frac{\left|-(3 n-3)+\left(3 e^{i \theta}-e^{3 i \theta}\right)\right|}{\left|3 e^{i \theta}-e^{3 i \theta}\right|}\left|a_{n}\right| \\
& \geq a_{1}-\sum_{n=2}^{\infty} \frac{3 n-1}{2}\left|a_{n}\right| \geq 0,
\end{aligned}
$$

if the inequality (2.11) holds. Hence, the desired proof is completed.

By making use of the well-known Alexander relation between starlike and convex functions and in view of Theorem 2.1, following necessary and sufficient convolution conditions for the class $\mathcal{C}_{N e}$ are given.

Theorem 2.4. The function $f$ defined by (1.2) is in the class $\mathcal{C}_{N e}$ if and only if

$$
\frac{1}{z}\left[f(z) * \frac{z+[1-2 L] z^{2}}{(1-z)^{3}}\right] \neq 0
$$

for all $L=\frac{3+3 e^{i \theta}-e^{3 i \theta}}{3 e^{i \theta}-e^{3 i \theta}}$, where $\theta \in[0,2 \pi]$, and also $L=1$.

Reasoning along the similar lines as the proof of the Theorem 2.2 and Theorem 2.3, we establish following results for the class $\mathcal{C}_{N e}$. We are omitting the details.

Theorem 2.5. A necessary and sufficient condition for the function $f$ defined by (1.2) to be in the class $\mathcal{C}_{N e}$ is that

$$
a_{1}-\sum_{n=2}^{\infty} n \frac{3-3 n+3 e^{i \theta}-e^{3 i \theta}}{3 e^{i \theta}-e^{3 i \theta}} a_{n} z^{n-1} \neq 0 .
$$

Theorem 2.6. If the function $f$ defined by (1.2) satisfies the following inequality

$$
\sum_{n=2}^{\infty} n(3 n-1)\left|a_{n}\right| \leq 2 a_{1}
$$

then $f \in \mathcal{C}_{N e}$. 


\section{Quasi-Hadamard product properties}

In this section, we obtain quasi-Hadamard product of the classes $\mathcal{S}_{N e}^{*}$ and $\mathcal{C}_{N e}$. In order to prove further results in this section, we need to define a class $\mathcal{S}_{(c) N e}$ which as follows: A function $f$ of the form $(1.2)$ is in $\mathcal{S}_{(c) N e}$ if and only if the inequality

$$
\sum_{n=2}^{\infty} n^{c}(3 n-1) a_{n} \leq 2 a_{1}
$$

holds for any fixed non-negative real number $c$. It is noted that for $c=1, \mathcal{S}_{(1) N e} \equiv \mathcal{C}_{N e}$, and for $c=0, \mathcal{S}_{(0) N e} \equiv \mathcal{S}_{N e}^{*}$. Therefore for any positive integer $c$, following inclusion relation holds:

$$
\mathcal{S}_{(c) N e} \subset \mathcal{S}_{(c-1) N e} \subset \ldots \subset \mathcal{S}_{(2) N e} \subset \mathcal{C}_{N e} \subset \mathcal{S}_{N e}^{*} .
$$

Theorem 3.1. Let the functions $f_{i}$ defined by (1.3) be in the class $\mathcal{S}_{N e}^{*}$ for every $i=$ $1,2, \ldots m$. Then the quasi-Hadamard product $f_{1} * f_{2} * \ldots * f_{m}$ belongs to the class $\mathcal{S}_{(m-1) N e}$.

Proof. To prove the theorem, we need to show that

$$
\sum_{n=2}^{\infty}\left[n^{m-1}(3 n-1) \prod_{i=1}^{m} a_{n, i}\right] \leq 2 \prod_{i=1}^{m} a_{1, i} .
$$

Since $f_{i} \in \mathcal{S}_{N e}^{*}$, we have

$$
\sum_{n=2}^{\infty}(3 n-1) a_{n, i} \leq 2 a_{1, i}
$$

for every $i=1,2, \ldots m$. Thus,

$$
(3 n-1) a_{n, i} \leq 2 a_{1, i}
$$

or

$$
a_{n, i} \leq \frac{2}{(3 n-1)} a_{1, i}
$$

for every $i=1,2, \ldots m$. Since $\frac{3 n-1}{2}>n$ for every $n \geq 2$, thus $\frac{2}{3 n-1}<\frac{1}{n}$. Hence, the right side of the last inequality not greater than $n^{-1} a_{1, i}$. Thus, we obtain

$$
a_{n, i} \leq n^{-1} a_{1, i} \text {. }
$$

By making use of the inequality (3.2) for $i=1,2, \ldots m-1$ and the inequality (3.1) for $i=m$, we get

$$
\begin{aligned}
\sum_{n=2}^{\infty}\left[n^{m-1}(3 n-1) \prod_{i=1}^{m} a_{n, i}\right] & \leq \sum_{n=2}^{\infty}\left[n^{m-1}(3 n-1) a_{n, m}\left\{n^{-(m-1)} \prod_{i=1}^{m-1} a_{1, i}\right\}\right] \\
& =\sum_{n=2}^{\infty}(3 n-1) a_{n, m}\left\{\prod_{i=1}^{m-1} a_{1, i}\right\} \\
& \leq 2 \prod_{i=1}^{m} a_{1, i} .
\end{aligned}
$$

Since $\mathcal{S}_{(m-1) N e} \subset \mathcal{S}_{(m-2) N e} \subset \ldots \subset \mathcal{S}_{(0) N e} \equiv \mathcal{S}_{N e}^{*}$ and therefore, $f_{1} * f_{2} * \ldots * f_{m} \in \mathcal{S}_{(m-1) N e}$. This completes the proof.

Theorem 3.2. Let the functions $f_{i}$ defined by (1.3) be in the class $\mathcal{C}_{N e}$ for every $i=$ $1,2, \ldots m$. Then the quasi-Hadamard product $f_{1} * f_{2} * \ldots * f_{m}$ belongs to the class $\mathcal{S}_{(2 m-1) N e}$.

Proof. To prove the theorem, we need to show that

$$
\sum_{n=2}^{\infty}\left[n^{2 m-1}(3 n-1) \prod_{i=1}^{m} a_{n, i}\right] \leq 2 \prod_{i=1}^{m} a_{1, i}
$$


Since $f_{i} \in \mathcal{C}_{N e}$, we have

$$
\sum_{n=2}^{\infty} n(3 n-1) a_{n, i} \leq 2 a_{1, i}
$$

for every $i=1,2, \ldots m$. Thus

$$
n(3 n-1) a_{n, i} \leq 2 a_{1, i}
$$

or

$$
a_{n, i} \leq \frac{2}{n(3 n-1)} a_{1, i}
$$

for every $i=1,2, \ldots m$. Since $\frac{n(3 n-1)}{2}>n^{2}$ for every $n \geq 2$, thus $\frac{2}{n(3 n-1)}<\frac{1}{n^{2}}$. Then the right side of the last inequality not greater than $n^{-2} a_{1, i}$. Thus,

$$
a_{n, i} \leq n^{-2} a_{1, i}
$$

for every $i=1,2, \ldots m$. By making use of the inequality (3.4) for $i=1,2, \ldots m-1$ and the inequality (3.3) for $i=m$, we get

$$
\begin{aligned}
\sum_{n=2}^{\infty}\left[n^{2 m-1}(3 n-1) \prod_{i=1}^{m} a_{n, i}\right] & \leq \sum_{n=2}^{\infty}\left[n^{2 m-1}(3 n-1) a_{n, m}\left\{n^{-2(m-1)} \prod_{i=1}^{m-1} a_{1, i}\right\}\right] \\
& =\sum_{n=2}^{\infty} n(3 n-1) a_{n, m}\left\{\prod_{i=1}^{m-1} a_{1, i}\right\} \\
& \leq 2 \prod_{i=1}^{m} a_{1, i} .
\end{aligned}
$$

Since $\mathcal{S}_{(2 m-1) N e} \subset \mathcal{S}_{(2 m-2) N e} \subset \ldots \subset \mathcal{S}_{(1) N e} \equiv \mathcal{C}_{N e}$, thus, $f_{1} * f_{2} * \ldots * f_{m} \in \mathcal{S}_{(2 m-1) N e}$. This completes the proof.

Theorem 3.3. Let the functions $f_{i}$ defined by (1.3) be in the class $\mathcal{C}_{N e}$ for every $i=$ $1,2, \ldots m$; and let the functions $g_{j}$ defined by (1.4) be in the class $\mathcal{S}_{N e}^{*}$ for every $j=1,2, \ldots s$. Then the quasi-Hadamard product $f_{1} * f_{2} * \ldots * f_{m} * g_{1} * g_{2} * \ldots * g_{s}$ belongs to the class $\mathcal{S}_{(2 m+s-1) N e}$.

Proof. To prove the theorem, we need to show that

$$
\sum_{n=2}^{\infty}\left[n^{2 m+s-1}(3 n-1)\left\{\prod_{i=1}^{m} a_{n, i} \prod_{j=1}^{s} b_{n, j}\right\}\right] \leq 2\left\{\prod_{i=1}^{m} a_{1, i} \prod_{j=1}^{s} b_{1, j}\right\} .
$$

Since $f_{i} \in \mathcal{C}_{N e}$, we have

$$
\sum_{n=2}^{\infty} n(3 n-1) a_{n, i} \leq 2 a_{1, i}
$$

for every $i=1,2, \ldots m$, thus it is noted that

$$
n(3 n-1) a_{n, i} \leq 2 a_{1, i}
$$

or

$$
a_{n, i} \leq \frac{2}{n(3 n-1)} a_{1, i} .
$$

The right side of the last inequality not greater than $n^{-2} a_{1, i}$. Thus,

$$
a_{n, i} \leq n^{-2} a_{1, i}
$$

for every $i=1,2, \ldots m$. Similarly, since $g_{j} \in \mathcal{S}_{N e}^{*}$, we have

$$
\sum_{n=2}^{\infty}(3 n-1) b_{n, j} \leq 2 b_{1, j}
$$


for every $j=1,2, \ldots s$. Hence, we obtain

$$
b_{n, j} \leq n^{-1} b_{1, j}
$$

By using the inequality (3.5) for $i=1,2, \ldots m$, the inequality(3.7) for $j=1,2, \ldots s-1$ and the inequality (3.6) for $j=s$, we get

$$
\begin{aligned}
& \sum_{n=2}^{\infty}\left[n^{2 m+s-1}(3 n-1)\left\{\prod_{i=1}^{m} a_{n, i} \prod_{j=1}^{s} b_{n, j}\right\}\right] \\
& \leq \sum_{n=2}^{\infty}\left[n^{2 m+s-1}(3 n-1) b_{n, s}\left\{n^{-2 m} n^{-(s-1)} \prod_{i=1}^{m} a_{1, i} \prod_{j=1}^{s-1} b_{1, j}\right\}\right] \\
& \quad=\sum_{n=2}^{\infty}(3 n-1) b_{n, s}\left\{\prod_{i=1}^{m} a_{1, i} \prod_{j=1}^{s-1} b_{1, j}\right\} \\
& \leq 2\left\{\prod_{i=1}^{m} a_{1, i} \prod_{j=1}^{s} b_{1, j}\right\} .
\end{aligned}
$$

Since $\mathcal{S}_{(2 m+s-1) N e} \subset \mathcal{S}_{(2 m+s-2) N e} \subset \ldots \subset \mathcal{S}_{(2) N e} \subset \mathcal{C}_{N e} \subset \mathcal{S}_{N e}^{*}$, we conclude the required result.

\section{Third order Hermitian-Toeplitz and Hankel determinants}

The first result of this section provides the best possible lower and upper bounds for the Hermitian-Toeplitz determinants of third order for the class $\mathcal{S}_{N e}^{*}$. In order to prove this result, we need the following lemma due to Libera and Zlotkiewicz:

Lemma 4.1. [27, Lemma 3, p. 254] Let $\mathcal{P}$ be the class of analytic functions having the Taylor series of the form

$$
p(z)=1+p_{1} z+p_{2} z^{2}+p_{3} z^{3}+\cdots
$$

satisfying the condition $\operatorname{Re}(p(z))>0(z \in \mathbb{D})$. Then

$$
2 p_{2}=p_{1}^{2}+\left(4-p_{1}^{2}\right) \xi
$$

for some $\xi \in \overline{\mathbb{D}}$.

Theorem 4.2. Let the function $f \in \mathcal{A}$ be in the class $\mathcal{S}_{N e}^{*}$. Then the best possible bounds on third order Hermitian-Toeplitz are given by

$$
-\frac{1}{4} \leq\left|T_{3,1}(f)\right| \leq 1
$$

Proof. Let the function $f \in \mathcal{S}_{N e}^{*}$. Then, we have $z f^{\prime}(z) / f(z)=1+w(z)-w^{3}(z) / 3$, where $w(z)=c_{1} z+c_{2} z^{2} \cdots \in \Omega$. Therefore, for some $p \in \mathcal{P}$ of the form (4.1), it is noted that

$$
\frac{z f^{\prime}(z)}{f(z)}=\frac{5(p(z))^{3}+15(p(z))^{2}+3 p(z)+1}{3(p(z)+1)^{3}} .
$$

On equating the coefficients of like power terms, we get

$$
a_{2}=\frac{p_{1}}{2} \quad \text { and } \quad a_{3}=\frac{p_{2}}{4} .
$$

In view of (4.3) and Lemma 4.1 , for some $\xi \in \overline{\mathbb{D}}$, we have

$$
\begin{aligned}
2 \operatorname{Re}\left(a_{2}^{2} \overline{a_{3}}\right) & =2 \operatorname{Re}\left(\frac{p_{1}^{2}}{4} \cdot \frac{1}{4} \overline{p_{2}}\right) \\
& =\frac{1}{16} p_{1}^{2}\left(p_{1}^{2}+\left(4-p_{1}^{2}\right) \operatorname{Re}(\bar{\xi})\right) \\
& =\frac{1}{16}\left(p_{1}^{4}+\left(4-p_{1}^{2}\right) p_{1}^{2} \operatorname{Re}(\bar{\xi})\right),
\end{aligned}
$$


and

$$
2\left|a_{2}\right|^{2}=\frac{1}{2} p_{1}^{2}
$$

$$
\begin{aligned}
\left|a_{3}\right|^{2} & =\left|\frac{1}{4}\left(p_{2}\right)\right|^{2} \\
& =\frac{1}{16}\left(p_{1}^{4}+\left(4-p_{1}^{2}\right)^{2}|\xi|^{2}+2\left(4-p_{1}^{2}\right) p_{1}^{2} \operatorname{Re}(\bar{\xi})\right) .
\end{aligned}
$$

In view of expressions (1.7), (4.4), (4.5) and (4.6), we have

$$
\begin{aligned}
\left|T_{3,1}(f)\right|: & =1+\frac{1}{64}\left(3 p_{1}^{4}-32 p_{1}^{2}-\left(4-p_{1}^{2}\right)^{2}|\xi|^{2}+2\left(4-p_{1}^{2}\right) p_{1}^{2} \operatorname{Re}(\bar{\xi})\right) \\
& =F\left(p_{1}^{2},|\xi|, \operatorname{Re}(\bar{\xi})\right) .
\end{aligned}
$$

Making use of inequality $-R e \xi \leq|\xi| \geq R e \xi$, above expression is written as

and

$$
\left.\left|T_{3,1}(f)\right|: \leq 1+\frac{1}{64}\left(3 x^{2}-32 x-(4-x)^{2} y^{2}+2(4-x) x y\right)\right)=F(x, y)
$$

$$
\left.\left|T_{3,1}(f)\right|: \geq 1+\frac{1}{64}\left(3 x^{2}-32 x-(4-x)^{2} y^{2}-2(4-x) x y\right)\right)=G(x, y),
$$

where $p^{2}=: x \in[0,4]$ and $|\xi|=: y \in[0,1]$. By making use of second derivative test for function of two variable, we note that $F(x, y)$ has no extreme point in the interior region of the rectangular domain $S=[0,4] \times[0,1]$. Therefore, the function $F(x, y)$ has maximum value on the boundary of domain $S$ that is 1 . In similar way, the function $G(x, y)$ has the minimum value in the domain $S$ that is $-1 / 4$. The analysis done on the functions $F$ and $G$ for getting extreme values gives the desired inequality. The upper and the lower bounds are sharp for the function $f_{u}$ and $f_{l}$, respectively, which are defined by

$$
\frac{z f_{u}^{\prime}(z)}{f_{u}(z)}=1+z^{3}-\frac{1}{3} z^{9} \quad \text { and } \quad \frac{z f_{l}^{\prime}(z)}{f_{l}(z)}=1+z-\frac{1}{3} z^{3} .
$$

Next, we provide non-sharp upper bounds on some Hankel determinants of third order for the functions in the class $\mathcal{S}_{N e}^{*}$. In order to prove results related to Hankel determinants, we need following lemmas.

Lemma 4.3. [1, Lemma 3, p. 66] Let the function $p \in \mathcal{P}, 0 \leq \beta \leq 1$ and $\beta(2 \beta-1) \leq \delta \leq \beta$. Then

$$
\left|p_{3}-2 \beta p_{1} p_{2}+\delta p_{1}^{3}\right| \leq 2
$$

Lemma 4.4. [33, Lemma 2.3, p. 507] Let $p \in \mathcal{P}$. Then for all $n, m \in \mathbb{N}$,

$$
\left|\mu p_{n} p_{m}-p_{m+n}\right| \leq \begin{cases}2, & 0 \leq \mu \leq 1 \\ 2|2 \mu-1|, & \text { elsewhere }\end{cases}
$$

If $0<\mu<1$, then the inequality is sharp for the function $p(z)=\left(1+z^{m+n}\right) /\left(1-z^{m+n}\right)$. In the other cases, the inequality is sharp for the function $p_{0}(z)=(1+z) /(1-z)$.

Lemma 4.5. [20] Let $p \in \mathcal{P}$. Then, for any real number $\mu$, the following holds:

$$
\left|\mu p_{3}-p_{1}^{3}\right| \leq \begin{cases}2|\mu-4|, & \mu \leq \frac{4}{3} \\ 2 \mu \sqrt{\frac{\mu}{\mu-1}}, & \mu>\frac{4}{3}\end{cases}
$$

The result is sharp. If $\mu \leq \frac{4}{3}$, equality holds for the function $p_{0}(z):=(1+z) /(1-z)$, and if $\mu>\frac{4}{3}$, then equality holds for the function

$$
p_{1}(z):=\frac{1-z^{2}}{z^{2}-2 \sqrt{\frac{\mu}{\mu-1}} z+1} .
$$


Theorem 4.6. Let the function $f \in \mathcal{A}$ be in the class $\mathcal{S}_{N e}^{*}$. Then,

(i) $\left|H_{3}^{(1)}(f)\right| \leq 0.925696$,

(ii) $\left|H_{3}^{(2)}(f)\right| \leq 1.6225$,

(iii) $\left|H_{3}^{(3)}(f)\right| \leq 1.34575$.

Proof. In view of (1.6), the third order Hankel determinants $H_{3}^{(1)}(f), H_{3}^{(2)}(f)$ and $H_{3}^{(3)}(f)$ for the functions $f \in \mathcal{A}$ are given by

$$
\begin{aligned}
& H_{3}^{(1)}(f)=a_{3}\left(a_{2} a_{4}-a_{3}^{2}\right)-a_{4}\left(a_{4}-a_{2} a_{3}\right)+a_{5}\left(a_{3}-a_{2}^{2}\right), \\
& H_{3}^{(2)}(f)=a_{2}\left(a_{2} a_{6}-a_{5}^{2}\right)-a_{3}\left(a_{3} a_{6}-a_{4} a_{5}\right)+a_{4}\left(a_{3} a_{5}-a_{4}^{2}\right), \\
& H_{3}^{(3)}(f)=a_{3}\left(a_{5} a_{7}-a_{6}^{2}\right)-a_{4}\left(a_{4} a_{7}-a_{5} a_{6}\right)+a_{5}\left(a_{4} a_{6}-a_{5}^{2}\right) .
\end{aligned}
$$

Since the function $f \in \mathcal{S}_{N e}^{*}$, then from expression (4.2), we have

$$
\begin{aligned}
\frac{z f^{\prime}(z)}{f(z)}=1 & +a_{2} z+\left(2 a_{3}-a_{2}^{2}\right) z^{2}+\left(a_{2}^{3}-3 a_{2} a_{3}+3 a_{4}\right) z^{3}+\left(-a_{2}^{4}+4 a_{2}^{2} a_{3}-4 a_{2} a_{4}\right. \\
& \left.-2 a_{3}^{2}+4 a_{5}\right) z^{4}+\left(a_{2}^{5}-5 a_{2}^{3} a_{3}+5 a_{2}^{2} a_{4}+5 a_{2} a_{3}^{2}-5 a_{2} a_{5}-5 a_{3} a_{4}+5 a_{6}\right) z^{5} \\
& +\left(-a_{2}^{6}+6 a_{2}^{4} a_{3}-6 a_{2}^{3} a_{4}-9 a_{2}^{2} a_{3}^{2}+6 a_{2}^{2} a_{5}+12 a_{2} a_{3} a_{4}-6 a_{2} a_{6}+2 a_{3}^{3}\right. \\
& \left.-6 a_{3} a_{5}-3 a_{4}^{2}+6 a_{7}\right) z^{6}+\cdots
\end{aligned}
$$

and

$$
\begin{aligned}
\frac{5(p(z))^{3}+15(p(z))^{2}+3 p(z)+1}{3(p(z)+1)^{3}}= & +\frac{p_{1} z}{2}+\left(\frac{p_{2}}{2}-\frac{p_{1}^{2}}{4}\right) z^{2}+\frac{1}{12}\left(p_{1}^{3}-6 p_{1} p_{2}+6 p_{3}\right) z^{3} \\
& +\frac{1}{4}\left(p_{1}^{2} p_{2}-2 p_{1} p_{3}-p_{2}^{2}+2 p_{4}\right) z^{4}+\frac{1}{32}\left(-p_{1}^{5}+8 p_{1}^{2} p_{3}\right. \\
& \left.+8 p_{1} p_{2}^{2}-16 p_{1} p_{4}-16 p_{2} p_{3}+16 p_{5}\right) z^{5}+\frac{1}{192}\left(7 p_{1}^{6}\right. \\
& -30 p_{1}^{4} p_{2}+48 p_{1}^{2} p_{4}+96 p_{1} p_{2} p_{3}-96 p_{1} p_{5}+16 p_{2}^{3} \\
& \left.-96 p_{2} p_{4}-48 p_{3}^{2}+96 p_{6}\right) z^{6}+\cdots
\end{aligned}
$$

On equating the coefficients of like power of $z$, we have

$$
\begin{aligned}
a_{4}= & \frac{1}{72}\left(-p_{1}^{3}-3 p_{1} p_{2}+12 p_{3}\right) \\
a_{5}= & \frac{1}{576}\left(5 p_{1}^{4}-12 p_{1}^{2} p_{2}-18 p_{2}^{2}-24 p_{1} p_{3}+72 p_{4}\right) \\
a_{6}= & \frac{1}{5760}\left(-27 p_{1}^{5}+160 p_{1}^{3} p_{2}-72 p_{1}^{2} p_{3}-336 p_{2} p_{3}-6 p_{1}\left(7 p_{2}^{2}+36 p_{4}\right)+576 p_{5}\right), \\
a_{7}= & \frac{1}{103680}\left(262 p_{1}^{6}-2235 p_{1}^{4} p 2+2352 p_{1}^{3} p_{3}+36 p_{1}^{2}\left(97 p_{2}^{2}-24 p_{4}\right)\right. \\
& \left.\quad-72 p_{1}\left(7 p_{2} p_{3}+48 p_{5}\right)+90\left(p_{2}^{3}-60 p_{2} p_{4}-32 p_{3}^{2}+96 p_{6}\right)\right)
\end{aligned}
$$

After rearrangement of terms and on applying triangle inequality, the expressions given by (4.11) and (4.12) are written as

$$
\left|a_{4}\right| \leq \frac{1}{6}\left|p_{3}-\frac{1}{4} p_{1} p_{2}-\frac{1}{12} p_{1}^{3}\right|
$$

and

$$
576\left|a_{5}\right| \leq\left. 12|| p_{1}\right|^{2}\left|\frac{5}{12} p_{1}^{2}-p_{2}\right|+74\left|-\frac{12}{37} p_{1} p_{3}+p_{4}\right|+18\left|p_{2}\right|^{2} .
$$


In view of the fact $\left|p_{n}\right| \leq 2$ and by making use of Lemma 4.3 and Lemma 4.4 in inequalities (4.15) and (4.16), respectively, we have

$$
\left|a_{4}\right| \leq \frac{1}{3} \quad \text { and } \quad\left|a_{5}\right| \leq \frac{79}{144} .
$$

(i) For the function $f \in \mathcal{S}_{N e}^{*}$, using (4.3), (4.11), (4.12), (4.13) and (4.8), we have

$$
\begin{aligned}
20736 H_{3}^{(1)}(f)= & -49 p_{1}^{6}+57 p_{1}^{4} p_{2}-198 p_{1}^{2} p_{2}^{2}-486 p_{2}^{3}+312 p_{1}^{3} p_{3} \\
& +936 p_{1} p_{2} p_{3}-576 p_{3}^{2}-648 p_{1}^{2} p_{4}+648 p_{2} p_{4} \\
= & 57 p_{1}^{4}\left(-\frac{49}{57} p_{1}^{2}+p_{2}\right)+936 p_{1} p_{2}\left(-\frac{11}{52} p_{1} p_{2}+p_{3}\right) \\
& +648 p_{4}\left(-p_{1}^{2}+p_{2}\right)+312 p_{3}\left(p_{1}^{3}-\frac{24}{13} p_{3}\right)-486 p_{2}^{3}
\end{aligned}
$$

By making use of triangle inequality, Lemmas 4.4, 4.5 and the fact $\left|p_{n}\right| \leq 2$, the expression (4.18) becomes

$$
\begin{aligned}
20736\left|H_{3}^{(1)}(f)\right| & \leq 57(2)^{5}+936(2)^{3}+648(2)^{2}+312(2)^{2}\left(\frac{24}{13}\right) \sqrt{\frac{24}{11}}+486(2)^{3} \\
& =15792+4608 \sqrt{\frac{6}{11}}
\end{aligned}
$$

which implies

$$
\left|H_{3}^{(1)}(f)\right| \leq \frac{329}{432}+\frac{2}{3} \sqrt{\frac{2}{33}} \approx 0.925696 .
$$

(ii) Further, if $f \in \mathcal{S}_{N e}^{*}$, using (4.3), (4.11), (4.12), (4.13) and (4.9), we have

$$
\begin{aligned}
29859840 H_{3}^{(2)}(f)= & -34992 p_{1}^{7}-1045 p_{1}^{9}+207360 p_{1}^{5} p_{2}+4320 p_{1}^{7} p_{2}-54432 p_{1}^{3} p_{2}^{2}+11448 p_{1}^{5} p_{2}^{2} \\
& -49680 p_{1}^{3} p_{2}^{2}+18468 p_{1} p_{2}^{4}-93312 p_{1}^{4} p_{3}+7920 p_{1}^{6} p_{3}-435456 p_{1}^{2} p_{2} p_{3} \\
& -12960 p_{1}^{4} p_{2} p_{3}-67392 p_{1}^{2} p_{2}^{2} p_{3}+31104 p_{2}^{2} p_{3}+8640 p_{1}^{3} p_{3}^{2}-138240 p_{3}^{3} \\
& -32400 p_{1}^{5} p_{4}+51840 p_{1}^{3} p_{2} p_{4}+108864 p_{1} p_{2}^{2} p_{4}+155520 p_{1}^{2} p_{3} p_{4} \\
& +311040 p_{2} p_{3} p_{4}-233280 p_{1} p_{4}^{2}+746496 p_{1}^{2} p_{5}-186624 p_{2}^{2} p_{5}-279936 p_{1}^{3} p_{4} .
\end{aligned}
$$

After rearrangement of terms and using triangle inequality, above expression can be written as

$$
\begin{aligned}
29859840\left|H_{3}^{(2)}(f)\right| \leq & 207360\left|p_{1}\right|^{5}\left|-\frac{27}{160} p_{1}^{2}+p_{2}\right|+4320\left|p_{1}\right|^{7}\left|-\frac{209}{864} p_{1}^{2}+p_{2}\right| \\
& +49680\left|p_{2}\right|^{2}\left|p_{1}\right|^{3}\left|\frac{53}{230} p_{1}^{2}-p_{2}\right|+12960\left|p_{1}\right|^{4}\left|p_{3}\right|\left|\frac{11}{18} p_{1}^{2}-p_{2}\right| \\
& +31104\left|p_{3}\right|\left|p_{2}\right|^{2}\left|-\frac{13}{6} p_{1}^{2}+p_{2}\right|+8640\left|p_{3}\right|^{2}\left|p_{1}^{3}-16 p_{3}\right| \\
& +746496\left|p_{1}\right|^{2}\left|-\frac{3}{8} p_{1} p_{4}+p_{5}\right|+51840\left|p_{1}\right|^{3}\left|p_{4}\right|\left|-\frac{5}{8} p_{1}^{2}+p_{2}\right| \\
& +186624\left|p_{2}\right|^{2}\left|\frac{7}{12} p_{1} p_{4}-p_{5}\right|+233280\left|p_{1}\right|\left|p_{4}\right|\left|\frac{2}{3} p_{1} p_{3}-p_{4}\right| \\
& +18468\left|p_{1}\right|\left|p_{2}\right|^{4}+311040\left|p_{2}\right|\left|p_{3}\right|\left|p_{4}\right|+93312\left|p_{1}\right|^{4}\left|p_{3}\right| \\
& +435456\left|p_{1}\right|^{2}\left|p_{2}\right|\left|p_{3}\right|+54432\left|p_{1}\right|^{3}\left|p_{2}\right|^{2} .
\end{aligned}
$$


Using Lemmas 4.4, 4.5 and the fact $\left|p_{n}\right| \leq 2$, above inequality becomes

$$
\begin{aligned}
29859840\left|H_{3}^{(2)}(f)\right| \leq & 207360(2)^{6}+4320(2)^{8}+54432(2)^{5}+49680(2)^{6}+18468(2)^{5} \\
& +12960(2)^{6}+93312(2)^{5}+31104(2)^{4}\left(\frac{10}{3}\right)+435456(2)^{4} \\
& +8640(2)^{3}(16) \sqrt{\frac{16}{15}}+746496(2)^{3}+51840(2)^{5} \\
& +186624(2)^{3}+233280(2)^{3}+311040(2)^{3} \\
= & 256(184787+1152 \sqrt{15}),
\end{aligned}
$$

which implies that

$$
\left|H_{3}^{(2)}(f)\right| \leq \frac{184787}{116640}+\frac{4}{27 \sqrt{15}} \approx 1.6225
$$

(iii) In view of (4.11), (4.12) and (4.13), a simple calculation yields

$$
\begin{aligned}
1658880\left(a_{4} a_{6}-a_{5}^{2}\right)= & -5\left(-5 p_{1}^{4}+12 p_{1}^{2} p_{2}+24 p_{1} p_{3}+18\left(p_{2}^{2}-4 p_{4}\right)\right)^{2} \\
& +4\left(p_{1}^{3}+3 p_{1} p_{2}-12 p_{3}\right)\left(27 p_{1}^{5}-160 p_{1}^{3} p_{2}+42 p_{1} p_{2}^{2}+72 p_{1}^{2} p_{3}\right. \\
& \left.+336 p_{2} p_{3}+216 p_{1} p_{4}-576 p_{5}\right) \\
= & -17 p_{1}^{8}+284 p_{1}^{6} p_{2}+192 p_{1}^{5} p_{3}-1572 p_{1}^{4} p_{2}^{2}-2736 p_{1}^{4} p_{4}+7008 p_{1}^{3} p_{2} p_{3} \\
& -2304 p_{1}^{3} p_{5}-1656 p_{1}^{2} p_{2}^{3}+11232 p_{1}^{2} p_{2} p_{4}-6336 p_{1}^{2} p_{3}^{2}-2304 p_{1} p_{2}^{2} p_{3} \\
& -6912 p_{1} p_{2} p_{5}+6912 p_{1} p_{3} p_{4}-1620 p_{2}^{4}+12960 p_{2}^{2} p_{4}-16128 p_{2} p_{3}^{2} \\
& +27648 p_{3} p_{5}-25920 p_{4}^{2} .
\end{aligned}
$$

On rearrangement of terms, above expression becomes

$$
\begin{aligned}
1658880\left(a_{4} a_{6}-a_{5}^{2}\right)= & 284 p_{1}^{6}\left(-\frac{17}{284} p_{1}^{2}+p_{2}\right)+27648 p_{5}\left(-\frac{1}{4} p_{1} p_{2}+p_{3}\right) \\
& +25920\left(\frac{4}{15} p_{1} p_{3}-p_{4}\right)+11232 p_{1}^{2} p_{2}\left(-\frac{23}{156} p_{2}^{2}+p_{4}\right) \\
& +12960 p_{2}^{2}\left(-\frac{581}{648} p_{2}^{2}+p_{4}\right)+2736 p_{1}^{4}\left(\frac{4}{57} p_{1} p_{3}-p_{4}\right) \\
& +7008 p_{1}^{3} p_{3}-2304 p_{1} p_{2}^{2} p_{3}-6336 p_{1}^{2} p_{3}^{2}-16128 p_{2} p_{3}^{2} \\
& -2304 p_{1}^{3} p_{5}-1572 p_{1}^{4} p_{2}^{2} .
\end{aligned}
$$

Using triangle inequality and Lemma 4.4 in (4.19), we get

$$
1658880\left|a_{4} a_{6}-a_{5}^{2}\right| \leq 1098432
$$

which implies

$$
\left|a_{4} a_{6}-a_{5}^{2}\right| \leq \frac{1907}{2880}
$$


In view of (4.11), (4.12), (4.13) and (4.14), a simple calculation yields

$$
\begin{aligned}
29859840\left(a_{4} a_{7}-a_{5} a_{6}\right)= & 167 p_{1}^{9}-4320 p_{1}^{7} p_{2}+576 p_{1}^{6} p_{3}+27648 p_{1}^{5} p_{2}^{2}+30672 p_{1}^{5} p_{4} \\
& -91584 p_{1}^{4} p_{2} p_{3}-12096 p_{1}^{4} p_{5}-20880 p_{1}^{3} p_{2}^{3}-95040 p_{1}^{3} p_{2} p_{4} \\
& +108864 p_{1}^{3} p_{3}^{2}-34560 p_{1}^{3} p_{6}+116640 p_{1}^{2} p_{2}^{2} p_{3}+103680 p_{1}^{2} p_{2} p_{5} \\
& -41472 p_{1}^{2} p_{3} p_{4}-7884 p_{1} p_{2}^{4}+57024 p_{1} p_{2}^{2} p_{4}-62208 p_{1} p_{2} p_{3}^{2} \\
& -103680 p_{1} p_{2} p_{6}-41472 p_{1} p_{3} p_{5}+139968 p_{1} p_{4}^{2}-50112 p_{2}^{3} p_{3} \\
& +93312 p_{2}^{2} p_{5}-41472 p_{2} p_{3} p_{4}-138240 p_{3}^{3}+414720 p_{3} p_{6} \\
& -373248 p_{4} p_{5} \\
= & 4320 p_{1}^{7}\left(\frac{167}{4320} p_{1}^{2}-p_{2}\right)+20880 p_{1}^{3} p_{2}^{2}\left(\frac{192}{145} p_{1}^{2}-p_{2}\right) \\
& +91584 p_{1}^{4} p_{3}\left(-\frac{1}{159} p_{1}^{2}+p_{2}\right)+108864 p_{3}^{2}\left(-\frac{80}{63} p_{3}+p_{1}^{3}\right) \\
& +62208 p_{1} p_{2} p_{3}\left(\frac{3645}{19444} p_{1} p_{2}-p_{3}\right)+93312 p_{2}^{2}\left(-\frac{29}{54} p_{2} p_{3}+p_{5}\right) \\
& -12096 p_{1}^{4}\left(\frac{1417}{56} p_{1} p_{4}-p_{5}\right)+41472 p_{2} p_{4}\left(\frac{11}{8} p_{1} p_{2}-p_{3}\right) \\
& +139968 p_{1} p_{4}\left(-\frac{8}{27} p_{1} p_{3}+p_{4}\right)+103680 p_{1} p_{2}\left(p_{1} p_{5}-p_{6}\right) \\
& +34560\left(12 p_{3}-p_{1}^{3}\right)-7884 p_{1} p_{2}^{4}-95040 p_{1}^{3} p_{2} p_{5} \\
& -41472 p_{1} p_{3} p_{5}-373248 p_{4} p_{5} .
\end{aligned}
$$

By making use of triangle inequality, Lemmas $4.4,4.5$ and the fact $\left|p_{n}\right| \leq 2$ in above inequality, we get

$$
29859840\left|a_{4} a_{7}-a_{5} a_{6}\right| \leq \frac{1152}{187}(6193253+48960 \sqrt{33}+42240 \sqrt{85})
$$

implies that

$$
\left|a_{4} a_{7}-a_{5} a_{6}\right| \leq \frac{4 \sqrt{\frac{5}{17}}}{27}+\frac{33119}{25920}+\frac{1}{3 \sqrt{33}}
$$


In view of (4.12), (4.13) and (4.14), a simple calculation yields

$$
\begin{aligned}
& 298598400\left(a_{5} a_{7}-a_{6}^{2}\right)=5\left(5 p_{1}^{4}-12 p_{1}^{2} p_{2}-24 p_{1} p_{3}-18 p_{2}^{2}+72 p_{4}\right)\left(262 p_{1}^{6}-2235 p_{1}^{4} p_{2}\right. \\
& +2352 p_{1}^{3} p_{3}+36 p_{1}^{2}\left(97 p_{2}^{2}-24 p_{4}\right)-72 p_{1}\left(7 p_{2} p_{3}+48 p_{5}\right) \\
& \left.+90\left(p_{2}^{3}-60 p_{2} p_{4}-32 p_{3}^{2}+96 p_{6}\right)\right)-9\left(27 p_{1}^{5}-160 p_{1}^{3} p_{2}\right. \\
& \left.+72 p_{1}^{2} p_{3}+42 p_{1} p_{2}^{2}+216 p_{1} p_{4}+336 p_{2} p_{3}-576 p_{5}\right)^{2} \\
= & -11 p_{1}^{10}+6165 p_{1}^{8} p_{2}-7632 p_{1}^{7} p_{3}-52992 p_{1}^{6} p_{2}^{2}-32256 p_{1}^{6} p_{4} \\
& +158544 p_{1}^{5} p_{2} p_{3}+193536 p_{1}^{5} p_{5}+114840 p_{1}^{4} p_{2}^{3}-265680 p_{1}^{4} p_{2} p_{4} \\
& -400896 p_{1}^{4} p_{3}^{2}+216000 p_{1}^{4} p_{6}+312768 p_{1}^{3} p_{2}^{2} p_{3}-1451520 p_{1}^{3} p_{2} p_{5} \\
& +670464 p_{1}^{3} p_{3} p_{4}-335556 p_{1}^{2} p_{2}^{4}+1495584 p_{1}^{2} p_{2}^{2} p_{4}-202176 p_{1}^{2} p_{2} p_{3}^{2} \\
& -518400 p_{1}^{2} p_{2} p_{6}+1161216 p_{1}^{2} p_{3} p_{5}-730944 p_{1}^{2} p_{4}^{2}-219456 p_{1} p_{2}^{3} p_{3} \\
& +746496 p_{1} p_{2}^{2} p_{5}-839808 p_{1} p_{2} p_{3} p_{4}+345600 p_{1} p_{3}^{3}-1036800 p_{1} p_{3} p_{6} \\
& +995328 p_{1} p_{4} p_{5}-8100 p_{2}^{5}+518400 p_{2}^{3} p_{4}-756864 p_{2}^{2} p_{3}^{2}-777600 p_{2}^{2} p_{6} \\
& +3483648 p_{2} p_{3} p_{5}-1944000 p_{2} p_{4}^{2}-1036800 p_{3}^{2} p_{4} \\
& +3110400 p_{4} p_{6}-2985984 p_{5}^{2} .
\end{aligned}
$$

In similar way, on rearrangement of terms and on applying triangle inequality, Lemmas 4.4, 4.5 and the fact $\left|p_{n}\right| \leq 2$ in above expression, we get

$$
\left|a_{5} a_{7}-a_{6}^{2}\right| \leq \frac{264619}{259200}
$$

On applying triangle inequality in (4.10), we have

$$
\left|H_{3}^{(3)}(f)\right| \leq\left|a_{3}\right|\left|a_{5} a_{7}-a_{6}^{2}\right|+\left|a_{4}\right|\left|a_{4} a_{7}-a_{5} a_{6}\right|+\left|a_{5}\right|\left|a_{4} a_{6}-a_{5}^{2}\right|
$$

and on putting the desired values from (4.17), (4.20), (4.21), (4.22) in above inequality, we have

$$
\left|H_{3}^{(3)}(f)\right| \leq \frac{4}{81} \sqrt{\frac{5}{17}}+\frac{8084743}{6220800}+\frac{1}{9 \sqrt{33}} \approx 1.34575
$$

\section{Acknowledgement}

The authors would like to express their gratitude to the referees for many valuable suggestions and insights that helped to improve quality and clarity of this manuscript.

\section{References}

[1] R.M. Ali, Coefficients of the inverse of strongly starlike functions, Bull. Malays. Math. Sci. Soc. 26 (1), 63-71, 2003.

[2] M.F. Ali, D.K. Thomas and A. Vasudevarao, Toeplitz determinants whose elements are the coefficients of analytic and univalent functions, Bull. Aust. Math. Soc. 97 (2), 253-264, 2018.

[3] M.K. Aouf, The quasi-Hadamard product of certain analytic functions, Appl. Math. Lett. 21, 1184-1187, 2008.

[4] K.O. Babalola, On $\mathrm{H}_{3}(1)$ Hankel determinant for some classes of univalent functions, Ineq. Theory and Appl. 6, 1-7, 2010.

[5] N. Breaz and R.M. El-Ashwah, Quasi-Hadamard product of some uniformly analytic and p-valent functions with negative coefficients, Carpathian J. Math. 30 (1), 39-45, 2014. 
[6] T. Bulboacă, M.K. Aouf and R.M. El-Ashwah, Convolution properties for subclasses of meromorphic univalent functions of complex order, Filomat 26 (1), 153-163, 2012.

[7] K. Cudna, O.S. Kwon, A. Lecko, Y.J. Sim and B. Śmiarowska, The second and thirdorder Hermitian Toeplitz determinants for starlike and convex functions of order $\alpha$, Bol. Soc. Mat. Mex. (3) 26 (2), 361-375, 2020.

[8] P.L. Duren, Univalent Functions, 259, Springer, New York, 1983.

[9] R.M. El-Ashwah, Some convolution and inclusion properties for subclasses of bounded univalent functions of complex order, Thai J. Math. 12 (2), 373-384, 2014.

[10] H. Güney, S. İlhan and J. Sokół, An upper bound for third Hankel determinant of starlike functions connected with k-Fibonacci numbers, Bol. Soc. Mat. Mex. (3) 25 (1), 117-129, 2019.

[11] W.K. Hayman, On the second Hankel determinant of mean univalent functions, Proc. London Math. Soc. (3) 18, 77-94, 1968.

[12] H.M. Hossen, Quasi-Hadamard product of certain p-valent functions, Demonstratio Math. 33 (2), 277-281, 2000.

[13] W. Janowski, Some extremal problems for certain families of analytic functions I, Ann. Polon. Math. 28, 297-326, 1973.

[14] P. Jastrzȩbski B. Kowalczyk, Oh S. Kwon, A. Lecko and Y.J. Sim, Hermitian Toeplitz determinants of the second and third-order for classes of close-to-star functions, Rev. R. Acad. Cienc. Exactas Fís. Nat. Ser. A Mat. RACSAM 114 (4), 166, 2020.

[15] W. Kaplan, Close-to-convex schlicht functions, Michigan Math. J. 1, 169-185, 1952.

[16] R. Kargar, A. Ebadian and J. Sokól, On Booth lemniscate and starlike functions, Anal. Math. Phys. 9 (1), 143-154, 2019.

[17] B. Kowalczyk, A. Lecko and Y.J. Sim, The sharp bound for the Hankel determinant of the third kind for convex functions, Bull. Aust. Math. Soc. 97 (3), 435-445, 2018.

[18] D. Kucerovsky, K. Mousavand and A. Sarraf, On some properties of Toeplitz matrices, Cogent Math. 3, 2016 (Article ID 1154705).

[19] V. Kumar, Hadamard product of certain starlike functions II, J. Math. Anal. Appl. 113, 230-234, 1986.

[20] V. Kumar, N. E. Cho, V. Ravichandran and H.M. Srivastava, Sharp coefficient bounds for starlike functions associated with the Bell numbers, Math. Slovaca 69 (5), 10531064, 2019.

[21] V. Kumar and S. Kumar, Bounds on Hermitian-Toeplitz and Hankel determinants for strongly starlike functions, Bol. Soc. Mat. Mex. (3) 27 (2), 1-16, 2021.

[22] V. Kumar, S. Kumar and V. Ravichandran, Third Hankel determinant for certain classes of analytic functions, in: International Conference on Recent Advances in Pure and Applied Mathematics, 223-231, Springer, Singapore, 2018.

[23] S. Kumar and V. Ravichandran, A subclass of starlike functions associated with a rational function, Southeast Asian Bull. Math. 40 (2), 199-212, 2016.

[24] O.S. Kwon, A. Lecko and Y.J. Sim, The bound of the Hankel determinant of the third kind for starlike functions, Bull. Malays. Math. Sci. Soc. 42 (2), 767-780, 2019.

[25] A. Lecko, Y.J. Sim and B. Śmiarowska, The sharp bound of the Hankel determinant of the third kind for starlike functions of order 1/2, Complex Anal. Oper. Theory 13 (5), 2231-2238, 2019.

[26] S.K. Lee, V. Ravichandran and S. Supramaniam, Bounds for the second Hankel determinant of certain univalent functions, J. Inequal. Appl. 2013 (1), 1-17, 2013.

[27] R.J. Libera and E.J. Złotkiewicz, Coefficient bounds for the inverse of a function with derivative in $\mathcal{P}$, Proc. Amer. Math. Soc. 87 (2), 251-257, 1983.

[28] W.C. Ma and D. Minda, A unified treatment of some special classes of univalent functions, in: Proceedings of the Conference on Complex Analysis, 157-169, International Press Inc., 1992. 
[29] P.T. Mocanu, Une propriété de convexité généralisée dans la théorie de la représentation conforme, Mathematica (Cluj) 34 (11), 127-133, 1969.

[30] S. Owa, On the classes of univalent functions with negative coefficients, Math. Japon, 27 (4), 409-416, 1982.

[31] Ch. Pommerenke, On the coefficients and Hankel determinants of univalent functions, J. London Math. Soc. 41, 111-122, 1966.

[32] Ch. Pommerenke, On the Hankel determinants of univalent functions, Mathematika 14, 108-112, 1967.

[33] V. Ravichandran and S. Verma, Bound for the fifth coefficient of certain starlike functions, C. R. Math. Acad. Sci. Paris, 353 (6), 505-510, 2015.

[34] M.O. Reade, On close-to-convex univalent functions, Michigan Math. J. 3, 59-62, 1955.

[35] M.S. Robertson, Certain classes of starlike functions, Michigan Math. J. 32 (2), 135$140,1985$.

[36] W. Rogosinski, Über positive harmonische Entwicklungen und typisch-reelle Potenzreihen, Math. Z. 35 (1), 93-121, 1932.

[37] K. Sharma, N.K. Jain and V. Ravichandran, Starlike functions associated with a cardioid, Afr. Mat. 27 (5-6), 923-939, 2016.

[38] J. Sokół, and J. Stankiewicz, Radius of convexity of some subclasses of strongly starlike functions, Zeszyty Nauk. Politech. Rzeszowskiej Mat. 19, 101-105, 1996.

[39] Y. Sun, Z.-G. Wang and A. Rasila, On third Hankel determinants for subclasses of analytic functions and close-to-convex harmonic mappings, Hacett. J. Math. Stat. 48 (6), 1695-1705, 2019

[40] L.A. Wani and A. Swaminathan, Starlike and convex functions associated with a nephroid domain, Bull. Malays. Math. Sci. Soc. 44 (1), 79-104, 2021.

[41] L.A. Wani and A. Swaminathan, Radius problems for functions associated with a nephroid domain, Rev. R. Acad. Cienc. Exactas Fís. Nat. Ser. A Mat. RACSAM 114 (4), 178, 2020.

[42] K. Ye and L.-H. Lim, Every matrix is a product of Toeplitz matrices, Found. Comput. Math. 16, 577-598, 2016.

[43] P. Zaprawa, Third Hankel determinants for subclasses of univalent functions, Mediterr. J. Math. 14 (1), 1-10, 2017. 